DOI: https://doi.org/10.46296/rc.v2i4.0008

\title{
El mercado virtual y el comportamiento de los compradores digitales.
}

\section{Virtual market and the behavoir of digital shoppers.}

\author{
Autora: Rivadeneira-Lucas Lilian Stefania \\ Universidad Laica Eloy Alfaro de Manabí (ULEAM). Manta, Ecuador. \\ e13116315660@live.uleam.edu.ec \\ Autora: Zambrano-Roldán Karen \\ Profesora Titular - Universidad Laica Eloy Alfaro de Manabí (ULEAM). Manta, Ecuador. \\ karen.zambranor339@gmail.com
}

\begin{abstract}
RESUMEN
El presente documento trata de forma generalizada sobre el comercio electrónico y el mercado virtual y como este influye en el comportamiento del consumidor, la evolución que ha tenido a lo largo de los años y los diferentes tipos de comercio electrónico para llegar al consumidor y la forma de promover los sitios de compras web como forma de comodidad del cliente y tener más ganancias.
\end{abstract}

Palabras claves: Comercio electrónico, mercado virtual, marketing digital, conducta del consumidor.

\begin{abstract}
This paper deals across the board with e-commerce and the virtual market and how it influences consumer behaviour, the evolution that it has had over the years and the different types of e-commerce to reach the consumer and how to promote web shopping sites as a form of customer convenience and make more profits.
\end{abstract}

Keywords: E-commerce, virtual market, digital marketing, consumer behavior.

Información del manuscrito:

Fecha de recepción: 09 de mayo de 2019.

Fecha de aceptación: 08 de julio de 2019.

Fecha de publicación: 10 de julio de 2019. 


\section{INTRODUCCIÓN}

Actualmente el mundo vive una época en la que se generan cambios y una continua evolución. El avance de las Tecnologías de la Información y Comunicación (TIC) ha llevado a que nuestra vida cotidiana sea producto de un cambio profundo, en el cual nos pasamos o dedicamos un tiempo o parte significativo de nuestro tiempo conectados a la Red, en el lugar de donde estemos, con el beneficio mediante ordenador, Tablet o teléfono móvil. En la actualidad se dispone de ciertas Plataformas Web viables, siempre interactuando porque cada vez que podemos realizar más acciones en el entorno se desarrolla un mundo virtual, en el que no sólo navegamos, también realizamos nuestras actividades diarias. En este nuevo entorno virtual tenemos una comunicación constante con otras personas mediante la llegada de las Redes Sociales, donde podemos compartir inclusive contenidos multimedia y hasta poder disfrutar de juegos en línea en grupo, o bien ejecutar distintas Aplicaciones Web. También tenemos participación en sitios web de Creación Propia, como el diseño de sitios de tipo blog o álbumes fotográficos, pero también encontramos soluciones para nuestras necesidades. El comercio electrónico juega un papel importante en la vida de los internautas. La globalización en la Red ha permitido la apertura de negocios en todo el mundo durante las 24 horas del día, por lo que ha aumentado las posibilidades de éxito de nuestro negocio.

El desarrollo de las Tecnologías de la Informática y las Comunicaciones (TIC) y su aplicación en la sociedad modifican la vida social, cultural y económica del hombre (Oliván, 2016). La evolución de Internet potencia que el intercambio de información se realice de manera más rápida y eficiente; además, posibilita que los usuarios accedan de forma más fácil a bienes y servicios. Internet aumenta constantemente su nivel de penetración mundial, durante el 2017 el $51.8 \%$ de la población mundial era usuario de Internet según indicadores del sitio Internet World Stats (Llanes, Sala, \& Leiva, 2018)

(Picazo, Ramirez, \& Luna, 2014) y (Jones, Motta, \& Alderete, 2016) definen el comercio electrónico (CE) como el proceso de automatizar la comercialización de bienes y servicios, mediante la utilización de una aplicación informática para satisfacer las necesidades de los clientes y las empresas. El 
comercio electrónico puede ayudar a las organizaciones a realizar ahorros sustanciales de costos, aumentar los ingresos, proporcionar entregas más rápidas, reducir los costos de administración y mejorar el servicio al cliente (LIBU, BAHARI, \& NOORMINSHAH A. IAHAD, 2016).

A finales de la década de los años noventa, con el desarrollo de las tecnologías informáticas y la expansión de las telecomunicaciones, se fortaleció un proceso de globalización e interdependencia económicas, que vio el nacimiento de una forma novedosa de realizar actividades comerciales, y se creó un nuevo proceso de oferta y demanda, donde tanto oferentes como demandantes situaron sus transacciones a través de medios electrónicos, principalmente Internet; así surgieron las empresas digitales y los consumidores digitales, cuyas actividades dan vida al mercado virtual. El comercio llevado a cabo por medios electrónicos, si bien tiene sus orígenes en el comercio tradicional y adopta principios de la economía clásica, como el proceso de oferta, demanda, precio, entre otros, su naturaleza tecnológica le imprime particularidades que conllevan a estudiarlo desde sus procesos intrínsecos, y formularle principios y características propios, de tal forma que conduzcan a un sistema exitoso en cualquier ámbito que lo analice o lo ponga en función. Lo anterior es fundamental, incluso para definirlo, puesto que no podemos reducir el significado del comercio electrónico, a considerarlo sólo como una forma más de realizar actos de mercado vía medios electrónicos; en realidad es mucho más. Diversas y distintas son las áreas del conocimiento que estudian el comercio electrónico; cuando nos preguntamos en materia jurídica qué es éste, debemos considerar que no existe una definición única y precisa de él. Su ejecución va marcando las pautas para comprender cómo funciona y por qué debe establecerse un marco normativo. Aunque de forma simple, podemos definir al mercado virtual como la compraventa o intercambio de bienes o servicios a través de medios electrónicos. Existen diversas opiniones que consideran otros elementos que intervienen en el intercambio comercial (Biblioteca Jurídica Virtual del Instituto de Investigaciones Jurídicas de la UNAM , 2018). 


\section{METODOLOGÍA}

El objetivo primordial de este artículo es analizar los diferentes documentos relacionados con el mercado virtual, el comercio electrónico y como ha influenciado aquello en el consumidor.

Esta parte de documento se centra en la investigación de diversas fuentes acerca del comercio electrónico y la influencia que tiene este en los diferentes consumidores. Como el marketing digital ha influenciado en los consumidores el término "e - commerce" y lo que esto conlleva. Para ello se han utilizado diferentes fuentes bibliográficas como libros y tesis de años anteriores dedicadas al tema.

Se ha procedido a buscar información de tesis dedicadas al tema y a filtrar la información más importante, así como también se ha procedido a buscar información de diferentes autores para relacionarla con la información encontrada en las tesis. Al ser tan extensa la información encontrada, solo una parte de ella será parte de esta investigación.

\section{RESULTADOS}

\subsection{El comercio electrónico en el mundo.}

Para el año 2017, la población en el mundo es de 7.496 billones de habitantes, según las cifras de Population City, sitio web especializado en censos de población, donde se muestra un crecimiento ascendente (Population City, 2017)

Del total de esta población el $54 \%$ se encuentra urbanizada y tiene acceso a múltiples servicios como salud, educación, vivienda, alimentación, empleo y remuneración. Producto de esta combinación de posibilidades, los países han desarrollado sus industrias, regulaciones políticas y legales, así como sus actividades comerciales a nivel mundial.

Para ello, las empresas se han apoyado en los recursos humanos, financieros y tecnológicos, así como también en el trabajo constante de los 
gobiernos para encontrar las condiciones óptimas para el desarrollo económico de los países, acoplándose a las condiciones de los mercados mundiales actuales. La globalización ha sido un fenómeno que ha traído consigo mucho desarrollo en diferentes entornos, económicos, sociales, culturales, comerciales y principalmente tecnológicos, siendo el comercio electrónico uno de los más representativos en el mundo, ya que permite un importante cambio en la forma en que las empresas realizan sus negocios, en cómo estas difunden la información de sus productos y servicios, en la implementación de nuevos canales de distribución, con esto contribuye significativamente a la recuperación y crecimiento de la economía de los países que lo han adoptado (ALADI, 2016).

Para ello, las empresas se han apoyado en los recursos humanos, financieros y tecnológicos, así como también en el trabajo constante de los gobiernos para encontrar las condiciones óptimas para el desarrollo económico de los países, acoplándose a las condiciones de los mercados mundiales actuales. La globalización ha sido un fenómeno que ha traído consigo mucho desarrollo en diferentes entornos, económicos, sociales, culturales, comerciales y principalmente tecnológicos, siendo el comercio electrónico uno de los más representativos en el mundo, ya que permite un importante cambio en la forma en que las empresas realizan sus negocios, en cómo estas difunden la información de sus productos y servicios, en la implementación de nuevos canales de distribución, con esto contribuye significativamente a la recuperación y crecimiento de la economía de los países que lo han adoptado (ALADI, 2016).



Fuente: Ventas del comercio electrónico en todo el mundo, tomado de Emarketer 2019 (Emarketer, 2019) 
Los principales 10 países con un mayor nivel de ingresos de ventas en comercio electrónico se encuentra China, Estados Unidos de Norteamérica, Reino Unido, Japón, Alemania, Corea del Sur, Francia, Canadá, India y Brasil. Se puede evidenciar que los países asiáticos como China e India tienen un porcentaje de penetración de Internet menor de 60\%, mientras que el resto de los países que ocupan los primeros 10 lugares están por encima del 80\%.

Los países con mayor ingreso de ventas por región son:

- Asia: China, Japón, Corea del Sur, India e Indonesia

- Europa: Reino Unido, Alemania, Francia, España e Italia

- Medio Oriente: Arabia Saudita, Turquía e Israel

- Norteamérica: Estados Unidos, Canadá y México - Sudamérica: Brasil, Chile, Argentina, Colombia y Perú. (Linzán, 2019)

\subsection{El mercado virtual}

“El mercado virtual o electrónico se fundamenta en las nuevas tecnologías $y$, contrariamente al mercado convencional, permite que las transacciones comerciales se realicen sin importar el lugar físico donde se encuentren el comprador y el vendedor e, incluso, que la transacción se lleve a cabo en distintos momentos en el tiempo." (Melgarejo, 2016) En este mercado se produce el fenómeno de la "desgeografización", en el que no existen las fronteras entre los países: todos podemos contratar, el mercado se amplía. Ello genera una mayor demanda de los bienes o servicios y la reducción de los precios de estos. En otras palabras, se puede decir que el mercado se convierte en un mercado virtual, donde la distancia geográfica de las partes no impide la posibilidad de contratar. (Melgarejo, 2016)

\subsection{Internet y la mercadotecnia digital}

Una de las cuestiones importantes que deben considerarse al respecto es que la mercadotecnia digital no es realmente todo sobre tecnología; se trata de la gente. En este sentido es similar a la mercadotecnia tradicional: se trata de los especialistas en mercadotecnia (marketers) que conectan o enlazan a otras personas (consumidores) para preparar relaciones conducentes al ciclo ventascompra. La tecnología le facilita a los especialistas en mercadotecnia nuevas y 
desafiantes plataformas que le permiten la conexión con la gente en novedosas e innovadoras formas. La mercadotecnia digital no trata de entender a la tecnología de soporte, sino más bien cómo usan las personas la tecnología y la potencian para enganchar a sus clientes en mayor cantidad y con efectividad. Como se observa, se debe aprender a usar las herramientas de mercadotecnia digital que se tienen al alcance y, al mismo tiempo, entender a la gente que las usa para descifrar el potencial de venta. (Trejo, 2017)

\subsection{El comercio electrónico en Ecuador.}

"El Ecuador no ha tenido tanto éxito como Brasil en Latinoamérica ni como los grandes del comercio electrónico en el mundo, sin embargo, existe un gran potencial de crecimiento" (ALADI, 2016). En relación con el crecimiento de la población que al 2017 llega al total de 16,594.156 millones de habitantes, esta información indica que cada vez más personas tienen acceso a conectividad en el Ecuador. Según el estudio realizado por el Instituto Nacional de Estadísticas y Censos - INEC, en el año 2016 indica que ha aumentado en los cinco últimos años un $13.7 \%$ el acceso a equipamiento en lo que a computadoras portátiles se refiere, de igual forma las computadoras de escritorio han registrado un crecimiento de $0.3 \%$.

El director de la Cámara Ecuatoriana de Comercio Electrónico indica que en Ecuador este tipo de comercio genera, en promedio, alrededor de $\$ 540$ millones anuales con un ritmo de crecimiento de 35\% hasta 2015. Dentro del ranking de comercio electrónico en países de américa latina, Ecuador se encuentra ubicado en el octavo lugar de la lista. Como se puede observar Ecuador es uno de los países menos desarrollados en cuanto a comercio electrónico se refiere. Ecuador se posiciona entre los últimos lugares de Latinoamérica y es uno de los países que menor cantidad de ventas registradas por comercio electrónico. Sin embargo, esto significa que existe mucho potencial de crecimiento para Ecuador (ALADI, 2016).

En el Ecuador existe un ecosistema vivo y adecuado para el e-commerce, después de analizar cómo se ha venido desarrollando en los últimos años, el comercio electrónico es evidente la importancia de que las instituciones públicas 
y empresas privadas analicen la opción de participar en estos mercados a través de la creación de tiendas en línea como herramientas potenciadoras de sus ventas, potenciar visitas o incrementar las ventas en sus tiendas físicas (ALADI, 2016).

Por el momento, la mayor cantidad de transacciones se concentran en instituciones financieras o instituciones públicas que manejan plataformas de pagos de servicios, agendamiento de citas, atención a usuarios y ventas de servicios de estas (ALADI, 2016). Por otro lado, en un menor porcentaje se encuentran servicios de educación, compra de ropa, calzado, accesorios, compra de tickets aéreos y búsqueda de información de bienes y servicios en general.

Los usuarios ecuatorianos han visto como principal motivador para realizar compras en línea precios más accesibles, ahorro en tiempo y esfuerzo, la acumulación de puntos o beneficios para compras futuras, exclusividad y obligatoriedad (solo disponible en línea), facilidad, comodidad o simplemente han preferido este canal para realizar estas transacciones (Del Alcazar, 2015).

También es importante evidenciar la falta de oferta, ya que las compras, los ecuatorianos las realizan en páginas de otros países como Amazon, Alibaba, Aliexpress.com, Ebay.com. Antes de que el gobierno ecuatoriano pusiera algunas restricciones a la compra de bienes a través de webs de países del exterior, era muy común el uso de sistemas como el $4 \times 4$ para realizar transacciones de comercio electrónico y así acceder a productos en estas tiendas virtuales y, por la facilidad de utilizar empresas de envío de paquetes y mensajería estas compras llegaban a la puerta de su domicilio, llegando a representar casi un $89 \%$ de las compras online en Ecuador y de estas el 60\% fueron realizadas en Estados Unidos y Asia, provocando que la mayor cantidad de dinero generado por estas transacciones sea recaudado por eses países (Revista Lideres, 2016). 


\subsection{Factores que limitan o potencian el comercio en el Ecuador}

Las empresas y emprendedores que están interesados en participar en esta nueva forma de hacer negocios presentan algunos temores a la hora de plantearse la inversión en una tienda online como son los fraudes, complejidad técnica, los altos costos y falta de confianza (Shift LATAM, 2016). Además de lo señalado, también es importante identificar las condiciones que generan un entorno adecuado para que se desarrolle este tipo de comercio en el Ecuador como son:

- Las Entidades Financieras y las formas de pago.

- La infraestructura y logística.

- El Gobierno y sus regulaciones.

- Percepción de confianza.

- La Innovación.

El porcentaje restante de esas compras se realizan en plataformas como mercadolibre.com.ec, olx.com.ec, y otras compras de empresas que tienen sus propias tiendas virtuales en Ecuador como: yaesta.com, linio.com.ec, buenobinitobarato.ec, la bahía.ec y cadenas de retail como deprati.com.ec, eshoperx.com.ec y otras páginas que solamente son informativas y direccionan sus clientes a las tiendas. Hay datos que indican que la perspectiva en ese sector es muy amplia, en 2017, más del $50 \%$ de ventas en tiendas será influenciada por canales digitales, en 2018, el 70\% de comercio electrónico se enfocará en personalización de la experiencia y para el 2020, la inteligencia artificial será utilizada para reconocer al cliente y mejorar las ganancias hasta un 15\% (Del Alcazar, 2015).

\subsection{Ecosistema digital del e- commerce en el Ecuador}

Cuando se habla de ecosistema digital, se hace referencian a un conjunto de fenómenos industriales y con impacto económico que van de la mano de la adopción de Tecnologías de información y comunicación, es un ambiente creado a través de internet en donde puede potenciarse y darse a conocer las marcas frente a millones de clientes cibernéticos por medio de sus equipos tecnológicos. 
1. Conseguir más tráfico hacia un sitio web, esto se lo puede conseguir a través de varias estrategias que motiven al usuario a visitar frecuentemente la página, los medios más utilizados son promociones y programas de fidelización.

2. Lograr mayor interacción con los usuarios, también llamado "user engagement" que corresponde a una conexión entre la persona y el producto vinculado directamente a la Experiencia del Usuario creando fidelidad hacia la marca.

3. Fidelización de clientes, generar recompra del producto o servicio y que el cliente recomiende la marca.

4. Generar intensión de compra, que corresponde a la preferencia de la marca por encima de la competencia, no siempre corresponde a compras reales, se puede catalogar como intensión de compra a las reservas o cotizaciones solicitadas a través de los medios digitales, ya que no necesariamente incluye a la transacción a través de medios de pago (Herrera, 2014)

\subsection{Mercado para el comercio electrónico en el ecuador}

La demanda de bienes y servicios a través del comercio electrónico en el mundo crece a un ritmo imparable, donde el principal actor es el desarrollo tecnológico, el e-commerce se ha convertido en el método de compra preferido para muchos consumidores. (Huaman, 2016)

Esto se refleja en la facturación que, ha representado a los países con mayor presencia en este sector un crecimiento importante para sus economías. El comercio electrónico está revolucionando los sistemas de compra tradicionales que hasta ahora se conocía y avanza constantemente con la introducción de nuevas estrategias, técnicas y modelos de negocio que buscan optimizar la compra por internet y mejorar la experiencia de los clientes. Esta nueva forma de hacer negocios está permitiendo que los clientes puedan realizar sus compras desde el sofá de su casa, oficina, lugar de estudios, desde su bolsillo por medio de smarth phones y, ha podido integrar entregas rápidas para eliminar las filas de atención a clientes, las esperas para recibir información de bienes y servicios y, a estar solo a un clic de comprar, pagar y recibir los bienes 
y servicios que adquieren a través de este canal (ALADI, 2016). Los principales sectores que han encontrado una importante demanda en sector son las ventas retail online.

"Las compras online son una de las actividades más populares en el mundo, como lo muestra el resultado de las ventas en retail que alcanzan 1,55 billones de dólares en 2015, el 7,4\% de la facturación total en retail" (Gutierrez, 2016).

Para el 2016 según estudios de comercio electrónico "estima que las ventas online en retail supondrán el $8,7 \%$ sumando ambos canales, el online y el físico y que este crecimiento tendrá una proyección ascendente hasta alcanzar el $14,6 \%$ del mercado minorista a escala global en 2020" (Gutierrez, 2016).

\subsection{Segmentos a los que se dirige el comercio electrónico en el Ecuador}

En el Ecuador, según la Agencia de Regulación y Control de las Telecomunicaciones (ARCOTEL), hasta diciembre de 2016, el número de personas que están suscritas a servicios de internet fijo en el Ecuador suman $1,613,358$, estos equivalen al $9.80 \%$ de la población y las personas que cuentan con una suscripción a servicios de internet móvil son 7,774,484, equivalentes al 47.21\% de la misma (ARCOTEL, 2017) de un total "de 16,776.977 millones de habitantes, lo que indica una gran potencial de crecimiento" (Roger, 2015).

Con esta perspectiva el país pronostica gran demanda para el 2017 en este sector, la información disponible de la que se dispondrá será mucho mayor, lo que supone un mayor intercambio de datos entre empresas que permite la identificación de los segmentos a los que el comercio electrónico debe apuntar como engranaje indispensable para cubrir la demanda que existe en este sector (Roger, 2015)

Existen clases de segmentos a los que se dirige el comercio electrónico en el mundo y que son tendencia en países que están empezando a incursionar en este tipo de negocio como Ecuador y se encuentran clasificados en: 


\subsubsection{Empresa a empresa o B2B}

En este segmento el Ecuador, ha ganado terreno incluso antes de la aparición del comercio electrónico, los negocios de empresas con empresas es una actividad muy común en todos los sectores comerciales del mundo y también en el país. Las empresas dependen de los productos o servicios de otras empresas, uno de los principales actores que juega un papel preponderante en el desarrollo de un negocio son sus proveedores., son considerados como el punto de partida de toda la logística empresarial son de gran importancia dentro de la economía, es así que, en el año 2013 sus ventas ascendieron a USD 27.578,15 millones en relación a las 1.000 empresas más grandes del país, en este sector los ingresos provienen principalmente de empresas de comercio: 28\% manufactura; 14\% transporte, almacenamiento y comunicaciones; $6 \%$ explotación de minas y canteras; y, finalmente 5\% otros sectores, 100 proveedoras como representan el $36,07 \%$ de las ventas de ese grupo en el Ecuador (Revista EKOS, 2015).

\subsubsection{Negocio a consumidor o B2C}

Este es el segmento más conocido en el Ecuador y en el que se encuentran la mayoría de las empresas que están incursionando en este tipo de comercio, también conocido como business to consumer (negocio a consumidor). "Se lleva a cabo entre el negocio o (tienda virtual), y el usuario interesado en comprar productos o adquirir servicios" (Arellano, 2017).

En el país, este sector se ha enfocado en los clientes que tienen la posibilidad de acceder a los websites desde cualquier lugar principalmente a través de computadoras, pero cada vez son más los dispositivos electrónicos que permiten tener la accesibilidad para realizar compras de forma rápida y segura, utilizando canales de pago electrónicos, sean estos transferencias bancarias o tarjetas de crédito, aunque este último método aún no está bien desarrollado en el país, las empresas usan estos canales para potenciar sus ventas brindando mayor información de sus productos y servicios, también a través de ofertas y de política de descuentos en los precios para mejorar los canales de comercialización con sus clientes. 


\subsubsection{Comercio electrónico B2E}

En el Ecuador no todas las empresas crean condiciones laborales que les permitan potenciar las habilidades, crecimiento y estabilidad laboral para sus colaboradores, las grandes compañías están adoptando modelos internos para solventar necesidades de capacitación, formación, transacciones y procesos administrativos e incluso beneficios para la compra de productos y servicios desde su tienda online, a través de un intranet o portal de Internet, con el fin de que sus colaboradores se desarrollen adecuadamente en su entorno laboral y que esto genere mejor desempeño en sus funciones y produzca mayor rentabilidad para sus empresas, es de ahí que surge este tipo de comercio la relación comercial business to employee (negocio a empleado) se centra principalmente entre una empresa y sus empleados. Es otro sector que tiene un gran potencial en el país (ECU RED, 2017).

\subsubsection{Comercio electrónico C2C}

Plataformas como OLX, Mercado libre.com, han ingresado al Ecuador y son algunos de los ejemplos que permiten identificar esa forma de hacer negocio, donde una persona que ya no utiliza algún producto y busca ofrecerlo - venderlo, puede utilizarlas como medio para realizar esta transacción con otro consumidor (Arellano, 2017).

\subsubsection{Comercio electrónico G2C}

En el Ecuador estos segmentos han tenido un desarrollo, ya que por la ampliación del estado se han realizado algunas implementaciones que están permitiendo automatizar ciertos servicios como el SRI, el Servicio de Rentas internas ha creado una plataforma donde los usuarios pueden subir su información tributaria, generar tramites en línea, sus comprobantes de pago, atención a usuarios, etc. (Arellano, 2017) 


\subsubsection{Comercio electrónico B2G}

El comercio electrónico entre empresas y gobierno también ha tenido un desarrollo importante en el país, principalmente en lo que refiere a concursos o licitaciones para proyectos en las diferentes áreas productivas, manejando de forma transparente la asignación de contratos con el estado, el SERCOP (Servicio Nacional de Contratación Pública) es quien hoy por hoy maneja la asignación de ofertas. "El comercio electrónico no solo son compras en tiendas online, va más allá y se adentra a situaciones más complejas, como a estos modelos de negocios" (Arellano, 2017).

\subsection{Características del mercado artesanal, industrializado, electrónico}

\begin{tabular}{|c|c|c|c|}
\hline Características & Mercado artesanal & $\begin{array}{c}\text { Mercado } \\
\text { industrializado }\end{array}$ & Mercado electrónico \\
\hline $\begin{array}{l}\text { Referentes de } \\
\text { compra }\end{array}$ & $\begin{array}{l}\text { Productor y producto. } \\
\text { Es importante la } \\
\text { confianza en el } \\
\text { artesano, así como el } \\
\text { resultado de su } \\
\text { trabajo. }\end{array}$ & $\begin{array}{l}\text { Producto. La empresa } \\
\text { no se ofrece como } \\
\text { referente de compra o } \\
\text { lo hace por detrás del } \\
\text { producto. }\end{array}$ & $\begin{array}{l}\text { Productor y producto. El } \\
\text { productor necesita primero la } \\
\text { atención y confianza del } \\
\text { cliente para realizar la venta. } \\
\text { La posterior experiencia con el } \\
\text { producto o servicio supone } \\
\text { una segunda fase. }\end{array}$ \\
\hline $\begin{array}{l}\text { Producto o } \\
\text { servicio }\end{array}$ & $\begin{array}{l}\text { Totalmente } \\
\text { adecuado al al } \\
\text { comprador por la } \\
\text { capacidad de } \\
\text { interacción con éste } \\
\text { para la definición y } \\
\text { producción. Producto } \\
\text { personalizado }\end{array}$ & $\begin{array}{l}\text { Las economías de } \\
\text { escala promueven la } \\
\text { estandarización de } \\
\text { productos. }\end{array}$ & $\begin{array}{l}\text { La personalización de } \\
\text { productos de forma masiva se } \\
\text { consigue por la automatización } \\
\text { y robotización de procesos, así } \\
\text { como por las posibilidades de } \\
\text { interacción con el comprador y } \\
\text { la gestión de la información } \\
\text { sobre los clientes. }\end{array}$ \\
\hline
\end{tabular}




\begin{tabular}{|c|c|c|c|}
\hline Precio & $\begin{array}{l}\text { La personalización } \\
\text { de productos de } \\
\text { forma masiva se } \\
\text { consigue por la } \\
\text { automatización y } \\
\text { robotización de } \\
\text { procesos, así como } \\
\text { por las posibilidades } \\
\text { de interacción con el } \\
\text { comprador y la la } \\
\text { gestión de la } \\
\text { información sobre los } \\
\text { clientes. }\end{array}$ & $\begin{array}{lr}\text { Precios fijos } \quad y \\
\text { sometidos } & a \\
\text { necesidades del canal. }\end{array}$ & $\begin{array}{l}\text { Flexible, según necesidades } \\
\text { de productor y comprador. }\end{array}$ \\
\hline Distribución & Sin intermediación. & $\begin{array}{l}\text { Intermediación. El } \\
\text { intermediario aporta } \\
\text { diferentes valores. }\end{array}$ & $\begin{array}{l}\text { Desintermediación, sobre } \\
\text { todo, en productos y servicios } \\
\text { digitales. El intermediario sólo } \\
\text { aporta transporte. }\end{array}$ \\
\hline Comunicación & $\begin{array}{l}\text { Personal, integrada e } \\
\text { interactiva. }\end{array}$ & $\begin{array}{l}\text { Masiva, diversificada } \\
\text { en canales y } \\
\text { unidireccional. }\end{array}$ & $\begin{array}{l}\text { Personal e interactiva gracias } \\
\text { a las posibilidades de la Red. } \\
\text { Integración progresiva de } \\
\text { canales con el desarrollo } \\
\text { tecnológico. }\end{array}$ \\
\hline Servicio & Inmediato. & $\begin{array}{ll}\text { Pendiente } & \text { de } \\
\text { disponibilidad. } & \end{array}$ & $\begin{array}{lcr}\text { Inmediato } & \text { gracias } & \text { al } \\
\text { autoservicio } & 0 & \text { la } \\
\text { automatización de respuestas. }\end{array}$ \\
\hline $\begin{array}{l}\text { Conocimiento } \\
\text { del cliente }\end{array}$ & $\begin{array}{l}\text { Personalizado } \\
\text { operativo. }\end{array}$ & \begin{tabular}{lrr} 
Masivo, $r$ & \multicolumn{2}{r}{ grandes } \\
segmentaciones. & Poco \\
operativo por $r$ su \\
tamaño y las \\
generalizaciones & que \\
inducen a error. &
\end{tabular} & $\begin{array}{l}\text { Personalizado gracias al } \\
\text { desarrollo de las bases de } \\
\text { datos. Operativo gracias a las } \\
\text { nuevas tecnologías. }\end{array}$ \\
\hline
\end{tabular}

Fuente: Etxebarria, 2004.

\subsection{El marketing digital como estrategia del E-commerce.}

El marketing digital se define como el empleo de Internet como fuente de publicidad y difusión con el fin de aumentar las ventas de los productos ofertados (Lora \& Segarra, 2013). (Stokes, 2011) plantea que el marketing digital impulsa la creación de demanda mediante el poder de Internet y constituye el proceso de comercialización de una marca a través de los canales digitales. Esta técnica de promoción representa un eslabón fundamental en la proliferación del comercio electrónico y en el desarrollo del e-business. 
Estas tecnologías desempeñan un papel importante en el intercambio, promoción y ventas de productos y servicios en la actualidad y su utilización es cada vez mayor. Además, el $22 \%$ de la población mundial empleó esta tecnología durante el 2017 según reporte del sitio We Are Social. Esta nueva forma de negocio representa indudablemente un elemento impulsor para el desarrollo económico del sector empresarial; así como para naciones desarrolladas y en vías de desarrollo (Moon, Sultana, Nur, \& Saifuzzaman, 2017).

Para mantener la atención del cliente, es necesario crear una sólida relación con él y ofrecer servicios que lo atraigan a visitar el sitio web con frecuencia y comprar productos y servicios. Debido a la relevancia de los negocios electrónicos para las empresas, es crucial llevar a cabo actividades exitosas de marketing digital. Para ello es necesario aplicar soluciones apropiadas de comercialización electrónica que garanticen el éxito sostenido del negocio (Gerrikagoitia, Castandera, Rebóna, \& Alzua-Sorzabala, 2014).

(Niranjanamurthy, Kavyashree, Jagannath, \& Chahar, 2013) identifica que la seguridad de los negocios electrónicos es uno de los componentes visibles que más afectan al usuario final durante sus interacciones económicas en el mercado digital. Garantizar la seguridad en las transacciones financieras entre clientes y proveedores, combatir el fraude y los correos electrónicos no deseados, constituyen elementos esenciales en los negocios electrónicos, que influyen positivamente en el marketing del negocio (Yazdanifard \& Hunn, 2014).

(Yazdanifard \& Hunn, 2014) realizan un estudio sobre las principales estrategias de marketing empleadas por el gigante comercial Alibaba. (Yazdanifard \& Hunn, 2014) determinan que el éxito comercial de esta empresa se debe fundamentalmente a que realiza una segmentación del mercado mediante la combinación de factores como el marketing de productos, precios, ubicación y promoción. La estrategia de marketing desarrollada por este gigante del comercio electrónico gira entorno de los clientes y proveedores, como elementos fundamentales de su existencia. (Yazdanifard \& Hunn, 2014) identifican la creación de canales de comunicación en tiempo real entre compradores y vendedores haciendo uso de foros, blogs, servicios de 
conversación y grupos de usuarios en el sitio web, como una de las estrategias de marketing fundamental del gigante comercial. Estos canales de comunicación garantizan una mejor retroalimentación con los clientes, elemento muy importante que reduce el comportamiento comercial deshonesto y asegura que las transacciones sean más transparentes y confiables.

El descuento para estimular las ventas y retener a los clientes es otro método de marketing que destacan (Yazdanifard \& Hunn, 2014), así como la aplicación de servicios de valor agregado que aumentan el precio del producto. Servicios como envío, entrega instantánea, garantías y otros, garantizan que el costo total de los productos con servicios adicionales es más alto que el costo de producción, lo que asegura la obtención de ganancias.

(Gerrikagoitia, Castandera, Rebóna, \& Alzua-Sorzabala, 2014) plantea que, de manera similar a las ventas reales, el proceso de venta basado en el comercio electrónico debe estar en concordancia con las necesidades del cliente antes, durante y después de la venta, esto requiere un conocimiento preciso de las preferencias del cliente. Los negocios electrónicos deben averiguar a quién, qué, cómo y cuándo referirse al visitante virtual. (Gerrikagoitia, Castandera, Rebóna, \& Alzua-Sorzabala, 2014) sugiere que los procesos inteligentes de emarketing deben tener presente las relaciones necesarias entre las tendencias de e-marketing y las técnicas de minería de datos. En su estudio (Gerrikagoitia, Castandera, Rebóna, \& Alzua-Sorzabala, 2014) asevera que el empleo de técnicas de minería de datos constituye una herramienta esencial para desarrollar estrategias de marketing personalizadas según las características de los clientes a partir del análisis de su comportamiento en el sitio del negocio.

\subsection{El nuevo consumidor digital}

"La revolución digital ha implicado importantes cambios sociales, culturales y psicológicos que han dado lugar a la aparición de un nuevo tipo de consumidor, con un estilo de vida diferente y hábitos de compra muy particulares" (Cortina, 2019). Conforme declara el Estudio Anual de e - Commerce de 2018 en España, siete de cada diez internautas compran a través de Internet, lo que 
supone 19,4 millones de españoles, comprendidos entre los 16 y los 65 años (IAB, Estudio anual de ecommerce, 2018).

\subsection{Ewom, fuente de información para el consumidor digital}

La definición de eWOM (boca-boca electrónico) ofrecida por (HennigThurau, P.Gwinner, Walsh, \& D.Gremler, 2004) es, "cualquier opinión positiva o negativa realizada por consumidores actuales, potenciales o pasados sobre un determinado producto o empresa, que es puesto a disposición de multitud de personas y organizaciones a través de Internet".

El eWOM ejerce una gran influencia sobre la elección de una marca, o por el contrario la no elección de esta. Los consumidores que son muy fieles a una marca son más propensos a expresar sus opiniones positivas sobre esta, e incluso se convierte en defensores de la empresa (Fernandez-Carus, 2014).

Es importante hacer una distinción entre el tradicional y el actual eWOM:EIWOM (Word of Mouth), se caracteriza por ser una comunicación cara a cara, existe un vínculo entre ambos, emisor y receptor, normalmente se realiza entre personas de la familia, amigos, compañeros de trabajo o personas cercanas a nuestro entorno (Arenas, Rondan, \& Ramirez, 2012), y "se sostiene en tres pilares fundamentales, y a la vez interrelacionados: la recomendación, la conversación y la confianza" (ZENITH, 2016).

En eWOM, la comunicación suele darse entre personas que tienen poca o ninguna relación entre ellos. Las opiniones y valoraciones suelen ser expuestas de forma escrita a través de foros, blogs o Redes Sociales (Arenas, Rondan, \& Ramirez, 2012).

\subsection{Webrooming vs showrooming}

El Webrooming (también conocido como efecto ROPO: Research Online, Purchase Offline) consiste en buscar los productos en Internet para luego adquirirlos en un establecimiento físico, accediendo también de este modo a la opinión y valoración de otros consumidores sobre los productos o servicios, factor crucial a la hora de tomar la decisión correcta antes de realizar una compra (NEO ATTACK, s.f.). 
El 55\% practica Webrooming para ver opiniones de otros consumidores y un 44\% para saber con antelación lo que comprarán en el establecimiento. Datos proporcionados por Euro monitor (PURO MARKETING, 2015).

En el caso del showrooming, seria todo lo contrario al efecto ROPO, se trata de un efecto muy ligado a la aparición de los Smartphones, conectados a Internet las 24 horas del día, "la conectividad móvil está cambiando los comportamientos de compra en las tiendas físicas, teniendo un alto impacto para el comercio" (EL RINCON DEL MARKETING, 2015)

\subsection{La importancia del servicio de atención al consumidor digital}

Debido a los cambios sociales, económicos y tecnológicos, se ha modificado la forma de hacer negocios de las empresas para poder ser rentables y competir con el resto. Hemos pasado de una economía basada en el producto a otra basada en los servicios, la información, la atención y en el conocimiento (Barullas, 2016).

Las empresas tienen un nuevo perfil de consumidor al que atender, los social media. Este es un cliente social que actúa de manera multicanal con las compañías que le proveen de productos y servicios. La relación de la empresacliente se establece antes de que este decida adquirir el producto o servicio, y esta relación se amplía más allá del momento de la compra. Este tipo de consumidor es activo, se convierte en el protagonista absoluto de la relación, es él quien decide qué, cómo, dónde y cuándo lo quiere. Está muy bien informado e influye sobre el resto de los consumidores a través de las redes y comunidades virtuales. Opina, valora y cuanta su experiencia, pero también se puede ver influido por los comentarios y opiniones de otros consumidores. Las empresas tienen que trabajar para desarrollar herramientas que les ayuden a comunicarse con éxito con este nuevo tipo de consumidor. La relación entre ambos debe ser bidireccional, fluida y continua (Suanya, García, Núñez, \& Salas, 2013)

\subsection{E-commerce a través de las redes sociales}

Las compras a través de las Redes Sociales siguen en aumento. Poder comprar directamente con un solo clic en nuestra cuenta de Facebook o Instagram utilizando nuestro Smartphone cada vez en más común, o también en 
aplicaciones como Pinterest. Una forma de consumo más extendida entre los jóvenes. Según el Estudio anual de Redes Sociales en 2018 de IAB, "se extiende el uso de Redes Sociales para buscar información de productos antes de realizar la compra: un 57\% declara hacerlo. El canal para hacerlo es principalmente Facebook e Instagram que gana peso respecto al año 2017 y un 38\% realiza comentarios, opina, expone sus problemas o dudas sobre sus compras por Internet en alguna Red Social". Pero a pesar de estos datos, solo el $18 \%$ de los encuestados realizan compras a través de las Redes Sociales. Esto contrasta con el objetivo principal de las Redes Sociales, "el principal uso por parte de los profesionales de las Redes Sociales recae en vender, seguido de la generación de branding y servicio de atención al cliente" (IAB, Estudio Anual de Redes Sociales 2018, 2018).

\subsection{El consumidor del futuro}

“El consumidor de 2030 será digital y más exigente en un entorno en el que la velocidad de entrega será la clave, según un informe de EAE Business School" (Alonso, 2019). Según este informe el consumidor online del futuro será el más preparado y el que tendrá el mejor criterio, al disponer de mayor cantidad de información para su elección. Debido a la rapidez con la cual los productos pueden ser clonados o copiados por la competencia, las empresas apostarán por las emociones de sus clientes para incentivar el consumo. En este sentido nos encontraremos un consumidor mucho más exigente y selectivo, con demanda de productos y servicios personalizados, por las que este estaría dispuesto a hacer un sacrificio económico mayor. Según el informe de EAE "El desarrollo del Big Data y del Smart Data permitirá la segmentación más allá incluso de lo individual y podrá concretarse en momentos del día, estados de ánimo, disposición y muchos más factores" (Alonso, 2019).

El Smartphone seguirá siendo la herramienta principal para realizar compras online, serán el centro de las transacciones comerciales y además las aplicaciones móviles tenderán hacia usos más colectivos. "Estarán presentes en todo tipo de dispositivos móviles y servirán para definir perfiles de usuarios que las marcas aprovecharán para proyectar sus ofertas con una gran eficiencia en 
su focalización”, afirma el profesor de EAE Business School, Eduardo Irastorza (BYSNESS EL PERIODICO, 2019)

El objetivo de la tienda física ya no será el de cerrar una compra sino conseguir la lealtad del cliente hacia la marca y con ello aumentar su fidelidad, ya que en un mundo con tanta competencia los clientes tienen la tendencia de cambiar una marca por otra sin que esto les ocasione un mayor coste. La creatividad de las compañías será fundamental, para crear experiencias que no son satisfechas por el comercio electrónico. El servicio de atención al cliente será un punto clave en la fidelidad del cliente, sea tanto en el comercio online como en el comercio tradicional. Sus opiniones y valoraciones necesitan ser escuchadas en todo momento por las compañías si quieren que estos confíen en ellas (BYSNESS EL PERIODICO, 2019). "Los asistentes virtuales y el comercio electrónico serán dos importantes bazas para jugar para competir con ventaja" (Bernal, 2019).

\section{CONCLUSIONES}

El comercio electrónico está jugando un papel fundamental en las vidas de los nuevos seres actualizados, aquellos que están pendientes de las redes sociales, ya que las redes sociales son el medio óptimo para que las tiendas físicas puedan promover sus tiendas virtuales y así llegar a cualquier tipo de personas. Aunque en la actualidad no todos confían en las tiendas virtuales porque piensan que es un engaño y que el dinero que van a pagar en algún artículo va a ir a otras personas y lo consideran una estafa. Pero no todos consideran a las tiendas virtuales así, mientras que otros compran sus artículos por medio de internet, ponemos de ejemplo cuando se requiere traer un artículo desde otro país. En los próximos años, el comercio electrónico será mucho mejor que ir a una tienda física, ya que se ahorrara tiempo y se pueden realizar otras actividades en el tiempo que duraría ir a una tienda física. Convencer al consumidor de que la tienda virtual es un medio óptimo de compras, será el mayor reto de las empresas y tiendas.

Para concluir, el comportamiento del consumidor se basa, en parte, a lo que dicen otras personas acerca de esos lugares, es decir, el "boca - boca" 
influirá en el comportamiento del consumidor y si ira o no a una tienda física. En él lo que se lleva del año 2019, las tiendas virtuales han tenido un gran apogeo, porque la pandemia ha provocado que gran parte de la población mundial este confinada en sus casas y eso significa que no podían salir de sus casas, por aquello, todas las tiendas y lugares de servicio y comida han creado sus tiendas virtuales, como medio de llegar a toda la población confinada y hacer llegar de una u otra forma lo que ofrecen. Casos como estos, influyen en que, en los próximos meses y años, el comercio electrónico siga creciendo a pasos agigantados.

\section{REFERENCIAS}

ALADI. (2016). Ecuador. Obtenido de www.aladi.org/nsfaladi/reuniones.nsf/ceb...ad/\$FILE/ecuador.doc

Alonso, N. (2019). El consumidor del futuro: exigente, sibarita y emocional. Obtenido de Economia Digital: https://www.economiadigital.es/finanzas-ymacro/el-consumidor-del-futuro-exigente-sibarita-yemocional_614597_102.html

Arellano, G. (2017). Comercio Electronico \#7. Obtenido de SCRIB: https://es.scribd.com/document/359297668/Comercio-Electronico-7

Arenas, J., Rondan, J., \& Ramirez, P. (2012). El ewon en las redes sociales. Harvard Deusto Marketing y ventas No 114, 78-82.

Barullas, J. (2016). El comportamiento del consumidor y las nuevas tendencias de consumo ante las TIC. Obtenido de Blog de economia y empresa: http://economia-empresa.blogs.uoc.edu/marqueting/consumidor-ytendencias-consumo-tic/

Bernal, I. (2019). Exigente, sibarita y rencoroso... Así será el consumidor en 2030". Obtenido de El correo: https://www.elcorreo.com/economia/tueconomia/consumidor-2030-20190402170020-nt.html

Biblioteca Jurídica Virtual del Instituto de Investigaciones Jurídicas de la UNAM . (2018). El comercio electronico y principios economicos - comerciales. Obtenido de Biblioteca Jurídica Virtual del Instituto de Investigaciones Jurídicas de la UNAM https://archivos.juridicas.unam.mx/www/bjv/libros/10/4667/4.pdf

BYSNESS EL PERIODICO. (2019). Así serán los consumidores dentro de 10 años. Obtenido de Bysnezz El Periodico: 
https://byzness.elperiodico.com/es/noticias/sostenibles/20190329/comoseran-los-consumidores-dentro-de-10-anos-7379895

Cortina, J. L. (2019). Asi es el nuevo cliente digital. Obtenido de El pais: https://retina.elpais.com/retina/2019/02/22/innovacion/1550819091_2751 81.html

Del Alcazar, J. P. (2015). Cifras, estadisticas y estado del E-commerce en Ecuador. Obtenido de https://blog.formaciongerencial.com/estado-del-ecommerce-en-ecuador/

ECU RED. (2017). B2E. Obtenido de https://www.ecured.cu/B2E

EL RINCON DEL MARKETING. (2015). Qué es el Showrooming y cómo hacerle frente. Obtenido de EL RINCON DEL MARKETING: http://www.elrincondemarketing.es/que-es-el-showrooming-y-comohacerle-frente/

Emarketer. (2019). Emarketer.com. Obtenido de Global Ecommerce 2019 Ecommerce Continues Strong Gains Amid Global Economic Uncertainty: https://www.emarketer.com/content/global-ecommerce-2019

Etxebarria, G. J. (Julio de 2004). Ebookcentral.proquest.com. Obtenido de Ebookcentral.proquest.com 'e-consumer' : El consumidor y los retos del nuevo mercado. : Retrieved from http://ebookcentral.proquest.com

Fernandez-Carus, L. (2014). El comportamiento del consumidor online". Obtenido de Universidad de Oviedo.

Gerrikagoitia, J. K., Castandera, I., Rebóna, F., \& Alzua-Sorzabala, A. (2014). New trends of Intelligent E-Marketing based on Web Mining for e-shops. International Conference on Strategic Innovative Marketing, 75 - 83.

Gutierrez, A. (2016). Las ventas online en retail abarcarán el 14,6\% de las ventas totales en 2020. Obtenido de Ecommerce News: https://ecommercenews.es/las-ventas-online-retail-abarcaran-146-las-ventas-totales-2020/

Hennig-Thurau, T., P.Gwinner, K., Walsh, G., \& D.Gremler, D. (2004). Electronic word-of-mouth via consumer-opinion platforms: What motivates consumers to articulate themselves on the Internet? Journal of Interactive Marketing, 38 - 52.

Herrera, F. (2014). ¿Qué es y para qué Sirve el Ecosistema Digital para mi Pyme? Obtenido de https://marketingenredesociales.com/wpcontent/cache/all/que-es-y-para-que-sirve-el-ecosistema-digital-para-mipyme.html/index.html

Huaman, V. (2016). La tablet. Obtenido de Software de Productividad I: http://softwareproductividadvictor.blogspot.com/ 
IAB. (2018). Estudio anual de ecommerce. Obtenido de IAB: https://iabspain.es/estudio/estudio-anual-de-ecommerce-2018/

IAB. (2018). Estudio Anual de Redes Sociales 2018. Obtenido de IAB: https://iabspain.es/estudio/estudio-anual-de-redes-sociales-2018/

Jones, C., Motta, J., \& Alderete, M. V. (2016). Gestión estratégica de tecnologías de información y comunicación y adopción del comercio electrónico en Mipymes de Córdoba, Argentina. Estudios Gerenciales, 4 - 13.

LIBU, D., BAHARI, M., \& NOORMINSHAH A. IAHAD, W. I. (2016). SYSTEMATIC LITERATURE REVIEW OF E-COMMERCE . Journal of Theoretical and Applied Information Technology, 422 - 438.

Linzán, M. G. (2019). El comercio electrónico e-commerce, análisis actual desde la perspectiva del consumidor en la ciudad de Guayaquil, provincia del Guayas y estrategias efectivas para su desarrollo (Sistema de posgrado maestría). Guayaquil-Ecuador: Universidad Catolica Santiago de Guayaquil.

Llanes, R. P., Sala, H. V., \& Leiva, I. R. (2018). Estrategias de comercio electrónico y marketing digital para pequeñas y medianas empresas. Obtenido de Scielo: http://scielo.sld.cu/scielo.php?script=sci_arttext\&pid=S222718992018000300014

Lora, I., \& Segarra, M. (2013). Estudio exploratorio del uso del E-Marketing como una estrategia para micro, pequeñas y medianas empresas de servicios. Puente: Revista Científica de la Universidad Pontificia Bolivariana., 29-37.

Melgarejo, P. N. (2016). El comercio electrónico y la contratación electrónica: Bases del mercado virtual. Foro Jurídico Pontificia Universidad Católica del Perú, 59.

Moon, N. N., Sultana, S., Nur, F. N., \& Saifuzzaman, M. (2017). A Literature Review of the Trend of Electronic Commerce in Bangladesh Perspective. Global Journal of Management and Business Research: BEconomics and Commerce, $11-17$.

NEO ATTACK. (s.f.). Concepto de Webrooming. Obtenido de NEO ATTACK: https://neoattack.com/neowiki/webrooming/\#mas-informacion

Niranjanamurthy, M., Kavyashree, N., Jagannath, S., \& Chahar, D. (2013). Analysis of E-Commerce and M-Commerce: Advantages, Limitations and Security issues . International Journal of Advanced Research in Computer and Communication Engineeri, 2360-2370.

Picazo, S., Ramirez, P., \& Luna, L. F. (2014). Comercio electrónico y emprendimiento: un análisis aplicando la teoría del comportamiento 
planeado. RECAI: Revista de Estudios de Contaduria, Administracion e Informatica, 1 - 20.

Population City. (2017). Mundo · Población 1800-2100. Obtenido de Population City: http://poblacion.population.city/world/

Puro Marketing. (2016). Ecommerce en China: las sorprendentes cifras que demuestran su potencial. Obtenido de https://www.puromarketing.com/76/19782/ecommerce-chinasorprendentes-cifras-demuestran-potencial.html

Revista EKOS. (2015). Top 100 Proveedores business to business. Obtenido de Revista EKOS: http://www.ekosnegocios.com/negocios/verArticuloContenido.aspx?idArt $=5229$

Revista Lideres. (2016). El 'e-commerce' dinamiza el intercambio de bienes en el país. Obtenido de https://www.revistalideres.ec/lideres/ecommercetecnologia-internet-ecuador-ventas.html

Roger, J. (2015). Best Marketing. Obtenido de https://documents.tips/marketing/marketing-estrategico55b0f4d8b2b40.html

Shift LATAM. (2016). Situacion del ecommerce en Ecuador. Obtenido de http://shiftlatam.com/resumen-ecommerce-day-ecuador-2016/

Stokes, R. (2011). Emarketing: The Essential Guide to Digital Marketing 4TH edition. Quirk Education.

Suanya, O., García, m., Núñez, R., \& Salas, N. (2013). El cliente social: retos de la atención al cliente en el Universo de las Redes Sociales". Profit Editorial.

Trejo, J. M. (2017). Una descripción de las herramientas que apoyan la planeación estratégica de toda innovación de campaña web. En J. M. Trejo, Mercadotecnia digital (pág. 9). Mexico: Grupo Editorial Patria.

Yazdanifard, R., \& Hunn, M. T. (2014). The Review of Alibaba's Online Business Marketing Strategies Which Navigate them to Present Success. Global Journal of Management and Business Research: EMarketing, 33 - 40.

ZENITH. (2016). "¿Qué es y en qué consiste el Word-of-mouth?". Obtenido de BlogginZennith: https://blogginzenith.zenithmedia.es/que-es-y-en-queconsiste-el-word-mouth-diczionario/ 